\title{
Primeiro registro de Nyssomyia yuilli yuilli (Young \& Porter) e de Trichopygomyia longispina (Mangabeira) (Diptera: Psychodidae) no estado do Espírito Santo, Brasil
}

\author{
Israel de Souza Pinto ${ }^{1,2,3}$; Jeferson Gonçalves Pires $^{1}$; Claudiney Biral dos Santos ${ }^{1}$; \\ Thieres Marassati das Virgens ${ }^{1}$; Gustavo Rocha Leite ${ }^{1}$; Adelson Luis Ferreira ${ }^{1}$; Aloísio Falqueto ${ }^{1}$ \\ ${ }^{1}$ Laboratório de Parasitologia, Departamento de Patologia, Centro Biomédico, \\ Universidade Federal do Espírito Santo - UFES, \\ Av. Marechal Campos, 1468, CEP 29040-090, Vitória, ES, Brasil \\ ${ }_{2}^{2}$ Programa de Pós-graduação em Biologia Animal, Universidade Federal do Espírito Santo - UFES, \\ Av. Marechal Campos, 1468, CEP 29040-090, Vitória, ES, Brasil \\ ${ }^{3}$ Autor para correspondência: Israel de Souza Pinto, e-mail: pintoisrael@gmail.com
}

Pinto, I. S.; Pires, J. G.; Santos, C. B.; Virgens, T. M.; Leite, G. R.; Ferreira, A. L.; Falqueto, A. First Record of Nyssomyia yuilli yuilli (Young \& Porter) and Trichopygomyia longispina (Mangabeira) (Diptera: Psychodidae) in the state of Espírito Santo, Brazil. Biota Neotrop., vol. 8, no. 1, Jan./Mar. 2008. Available from: <http://www.biotaneotropica.org.br/v8n1/en/abstract?short-communication+bn00708012008>.

Abstract: The phlebotomine sand flies Nyssomyia yuilli yuilli (Young \& Porter) (Diptera: Psychodidae) and Trichopygomyia longispina (Mangabeira) (Diptera: Psychodidae) are recorded in the state of Espírito Santo, Brazil for the first time.

Keywords: sand flies, distribution, occurrence, Phlebotominae.

Pinto, I. S.; Pires, J. G.; Santos, C. B.; Virgens, T. M.; Leite, G. R.; Ferreira, A. L.; Falqueto, A. Primeiro registro de Nyssomyia yuilli yuilli (Young \& Porter) e de Trichopygomyia longispina (Mangabeira) (Diptera: Psychodidae) no estado do Espírito Santo, Brasil. Biota Neotrop., vol. 8, no. 1, jan./mar. 2008. Disponível em: $<$ http://www.biotaneotropica.org.br/v8n1/pt/abstract?short-communication+bn00708012008>.

Resumo: Os flebotomíneos Nyssomyia yuilli yuilli (Young \& Porter) (Diptera: Psychodidae) e Trichopygomyia longispina (Mangabeira) (Diptera: Psychodidae) são registrados pela primeira vez no estado do Espírito Santo, Brasil.

Palavras-chave: flebotomíneos, distribuição, ocorrência, Phlebotominae. 


\section{Introdução}

Os flebotomíneos (Diptera: Psychodidae, Phlebotominae) são reconhecidos pela sua importância para a saúde pública uma vez que diversas espécies desses insetos transmitem Leishmania ao homem. As principais ferramentas para a confecção de estratégias direcionadas de controle e de combate a esses vetores são o conhecimento da sua distribuição geográfica e temporal (Ibáñez-Bernal et al. 2004).

O estado do Espírito Santo, atualmente, apresenta registros de 55 espécies de flebotomíneos pertencentes aos gêneros: Bichromomyia, Brumptomyia, Evandromyia, Expapillata, Lutzomyia, Micropygomyia, Migonemyia, Nyssomyia, Pintomyia, Pressatia, Psathyromyia, Psychodopygus e Sciopemyia. Para o gênero Nyssomyia são registradas, nesse estado, duas espécies: $N$. intermedia e $N$. whitmani. (Martins et al. 1978, Young \& Duncan 1994, Ferreira et al. 2001, Galati 2003, Rangel \& Lainson 2003, Pinto \& Santos 2007).

Nyssomyia yuilli yuilli (Young \& Porter) tem sua distribuição conhecida para o Brasil, Bolívia, Colômbia, Equador, Peru e Venezuela. No Brasil, até o momento, foi encontrada nos estados do Amapá, Amazonas, Bahia, Mato Grosso, Pará, Rondônia e São Paulo (Young \& Duncan 1994). Essa espécie apresenta hábitos semidomésticos, sendo encontrada em troncos e copas de árvores, em plantações, em áreas marginais às florestas e abrigos de animais domésticos (Azevedo et al. 1996, Alexander et al. 2001, Rangel \& Lainson 2003).

Embora não esteja relacionada à transmissão de leishmanioses no Brasil, fêmeas de $N$. yuilli yuilli já foram encontradas naturalmente infectadas por formas flageladas semelhantes a Leishmania sp., no entanto o parasito não foi identificado com certeza (Arias et al. 1985). Na Colômbia, diferentemente, essa espécie apresenta importância na transmissão de Leishmania (Alexander et al. 2001).

Trichopygomyia longispina apresenta registros de ocorrência no Brasil, Colômbia, Guiana Francesa e Venezuela. No Brasil, já foi notificada nos estados do Amazonas, Bahia, Minas Gerais, Pará, Pernambuco, Rondônia, Roraima (Young \& Duncan 1994). Essa espécie parece estar relacionada a ecótopos de florestas úmidas como a Mata Atlântica e Floresta Amazônica. Isso é corroborado pela ausência dessa espécie na região centro-oeste do Brasil. Além disso, apresenta uma distribuição descontínua ao longo da costa brasileira e sua ausência em determinados estados deve estar relacionada ao nível de degradação da vegetação, já que esse inseto apresenta hábitos silvestres (Aguiar \& Medeiros 2003), o que é evidenciado pelos seus registros de captura em matas ou em domicílios localizados próximos a matas (Saraiva et al. 2006).

O presente trabalho tem por objetivo acrescentar N. yuilli yuilli e de T. longispina à fauna flebotomínica do estado do Espírito Santo.

\section{Material e Métodos}

Os flebotomíneos foram capturados na localidade de Roda D'água (20 $13,243^{\prime} \mathrm{S}$ e $\left.40^{\circ} 28,090^{\prime} \mathrm{W}\right)$, município de Cariacica, estado do Espírito Santo. Roda D'água é uma localidade de colonização antiga e endêmica para leishmaniose tegumentar americana, situada em um vale com altitudes variando de próximo ao nível do mar até 890 m, com resíduos de Mata Atlântica. Junto às residências, localizadas a menos de 100 metros das matas, cafezais e bananais representam o ambiente mais comum. O clima da região é tropical quente com índice de precipitação anual de $1500 \mathrm{~mm}$ (Feitoza 1986). Coletas adicionais foram realizadas, posteriormente, nas localidades de Pau Amarelo (20 16,293' S e $\left.40^{\circ} 32,485^{\prime} \mathrm{W}\right)$, Itaenga ( $19^{\circ} 50,701^{\prime} \mathrm{S}$ e $40^{\circ} 47,582^{\prime} \mathrm{W}$ ) e Jurama $\left(18^{\circ} 59,089^{\prime} \mathrm{S}\right.$ e $\left.40^{\circ} 14,529^{\prime} \mathrm{W}\right)$ pertencentes, respectivamente, aos municípios de Cariacica, Santa Teresa e Vila Valério.

As coletas foram realizadas nas três primeiras horas noturnas utilizando-se armadilhas luminosas CDC (Center of Disease Control), barracas de Shannon modificadas e capturador de sucção de Castro.
Os espécimes foram capturados em repouso no ambiente domiciliar por busca ativa em barraca de Shannon, em árvores, paredes de residências e anexos, pocilgas, canis, galinheiros e outros abrigos de animais domésticos. Os insetos capturados foram levados ao laboratório onde os flebotomíneos passaram por processo de triagem, sendo então montados em lâminas, segundo Barreto e Coutinho (1940) e identificados segundo critérios taxonômicos propostos por Galati (1995, 2003).

Os espécimes foram depositados na coleção do Centro de Referência Nacional e Internacional para Phlebotominae do Centro de Pesquisa René Rachou - FIOCRUZ, Belo Horizonte, Brasil.

\section{Resultados e Discussão}

Os exemplares Nyssomyia yuilli yuilli e de Trichopygomyia longispina foram capturados, inicialmente, na localidade de Roda D'água (20 $13,243^{\prime} \mathrm{S}$ e $\left.40^{\circ} 28,090^{\prime} \mathrm{W}\right)$, município de Cariacica, estado do Espírito Santo. Exemplares de N. yuilli yuilli foram, posteriormente, encontrados também nas localidades de Pau Amarelo (Cariacica), Itaenga (Santa Teresa) e Jurama (Vila Valério). Com o encontro de $N$. yuilli yuilli sobem para três o número de espécies pertencentes ao gênero Nyssomyia encontrados no Espírito Santo, sendo $N$. intermedia e $N$. whitmani, os outros dois representantes. Estes últimos são considerados os principais vetores de Leishmania (Viannia) braziliensis no Brasil e estudos de infecção natural N. yuilli yuilli são importantes para verificar uma possível participação desse inseto em um ciclo silvestre de Leishmania sp.

Trichopygomyia longispina tem sido encontrada freqüentemente em estudos da fauna flebotomínica em estados vizinhos (Andrade Filho et al. 1997, Saraiva et al. 2006) e o registro da espécie no estado do Espírito Santo foi tardio, possivelmente pelos hábitos silvestres e a baixa densidade do inseto. Esses registros de ocorrência aumentam a área de distribuição dessas espécies, além de ser o primeiro registro de ocorrência do gênero Trichopygomyia para o estado.

\section{Referências Bibliográficas}

AGUIAR, G.M. \& MEDEIROS, W.M. 2003. Distribuição regional e hábitats das espécies de flebotomíneos do Brasi. In Flebotomíneos do Brasil (E.F Rangel \& R Lainson, eds). Fiocruz, Rio de Janeiro, p.207-245.

ALEXANDER, B., AGUDELO, L.A., NAVARRO, F., RUIZ, F., MOLINA, J., AGUILERA, G., \& QUIÑONES, M.L. 2001. Phlebotominae sandflies and leishmaniasis risks in Colombian coffee plantations under two systems of cultivation. Med. Vet. Entomol. 15:364-373.

ANDRADE FILHO, J.D., CARNEIRO, A.P.S., LIMA, M.L.N., SANTIAGO, R.M., GAMA, M.A., SANTOS, C.A., FALCÃO, A.L. \& BRAZIL, R.P. 1997. Flebotomíneos de Timóteo, estado de Minas Gerais, Brasil (Diptera: Psychodidae). Cad. Saúde Publ. 13:767-770.

ARIAS, J.R., MILES, M.A., NAIFF, R.D., POVOA, M.M., FREITAS, R.A., BIANCARDI, C.B. \& Castellon, E.G. 1985. Flagellate infections of brazilian sand flies (Diptera: Psychodidae): isolation in vitro and biochemical identification of Endotrypanum and Leishmania. Am. J. Trop. Med. Hyg. 34:1098-1108.

AZEVEDO, A.C.R., VILELA, M.L., SOUZA, N.A., ANDRADE-COELHO, C.A., BARBOSA, A.F., FIRMO, A.L.S. \& RANGEL, E.F. 1996. The sand fly fauna (Diptera: Psychodidae, Phlebotominae) of a focus of cutaneous leishmaniasis in Ilhéus, state of Bahia, Brazil. Mem. Ins. Oswaldo Cruz 91:75-79.

BARRETO, M.P. \& COUTINHO, J.O. 1940. Processos de captura, dissecação e montagem de flebótomos. Ann. Fac. Med. São Paulo 16:173-187.

FEITOZA, L.R. 1986. Carta agroclimática do Espírito Santo. Governo do estado do Espírito Santo: Secretaria de Estado da Agricultura \& Empresa Capixaba de Pesquisa Agropecuária - EMCAPA.

FERREIRA, A.L., SESSA, P.A., VAREJÃO, J.B.M. \& FALQUETO, A. 2001. Distribution of sand flies (Diptera: Psychodidae) at different altitudes in 
an endemic region of american cutaneous leishmaniasis in the state of Espírito Santo, Brazil. Mem. Ins. Oswaldo Cruz 96:1061-1067.

GALATI, E.A.B. 1995. Phylogenetic systematics of Phlebotominae (Diptera, Psychodidae) with emphasis on American groups. Bol Dir Malariol San Amb 35(1):133-142.

GALATI, E.A.B. 2003. Classificação de Phlebotominae. In Flebotomíneos do Brasil (E.F Rangel \& R Lainson, eds). Fiocruz, Rio de Janeiro, p.23-51.

IBAÑEZ-BERNAL, S., RODRÍGUEZ-DOMÍNGUEZ, G., GÓMEZHERNÁNDEZ, C.H. \& RICARDEZ-ESQUINCA, J.R. 2004. First record of Lutzomyia evansi (Nuñez-Tovar 1924) in México (Diptera: Psychodidae, Phlebotominae). Mem. Ins. Oswaldo Cruz 99:127-129.

MARTINS, A.V., WILLIAMS, P. \& FALCÃO, A.L. 1978. American sand flies (Diptera: Psychodidae, Phlebotominae). Rio de Janeiro, Academia Brasileira de Ciências, 195p.

PINTO, I.S. \& SANTOS, C.B. 2007. Description of Lutzomyia (Lutzomyia) falquetoi sp nov. (Diptera: Psychodidae, Phlebotominae) a new species from the state of Espírito Santo, Brazil. 2007. Mem. Ins. Oswaldo Cruz 102(2):165-167.

RANGEL, E.F. \& LAINSON, R. 2003. (eds). 2003. Flebotomíneos do Brasil. Rio de Janeiro, Editora Fiocruz, 368p.

SARAIVA, L., LOPES, J.S., OLIVEIRA, G.B.M., BATISTA, F.A., FALCÃO, A.L. \& ANDRADE FILHO, J.D. 2006. Estudo dos flebotomíneos (Diptera: Psychodidae) em área de leishmaniose tegumentar americana nos municípios de Alto Caparaó e Caparaó, Estado de Minas Gerais. Rev. Soc. Bras. Med. Trop. 39:56-63.

YOUNG, D.G. \& DUNCAN, M.A. 1994. Guide to the identification and geographic distribution of Lutzomyia sand flies in Mexico, the West Indies, Central and South America (Diptera: Psychodidae). Florida, Memoirs of the American Entomological Institute 54, Associated Publishers, 881p.

Recebido em 20/09/07 Versão Reformulada recebida em 29/11/07 Publicado em 01/01/08 